\title{
Industries, économie créatives et technologies d'information et de communication
}

\section{Philippe Bouquillion}

\section{(2) OpenEdition}

\section{Journals}

Édition électronique

URL : http://journals.openedition.org/ticetsociete/876

DOI : 10.4000/ticetsociete.876

Éditeur

Association ARTIC

Référence électronique

Philippe Bouquillion, «Industries, économie créatives et technologies d'information et de

communication », tic\&société [En ligne], Vol. 4, n² 2 | 2010, mis en ligne le 21 mars 2011, consulté le 19 avril 2019. URL : http://journals.openedition.org/ticetsociete/876 ; DOI : 10.4000/ticetsociete.876 
tic\&société - 4 (2), 2010

\title{
Industries, économie créatives et technologies d'information et de communication
}

\author{
Philippe BOUQUILLION \\ Université Paris 8 \\ CEMTI

\section{p.bouquillion@free.fr}

Philippe Bouquillon est professeur à l'Université Paris 8. II est responsable du programme "Les industries créatives: Pour une théorie critique ", financé par l'Agence nationale la recherche, ainsi que du programme "Les groupes industriels de la culture et de la communication en Europe et en Amérique du Nord ", programme financé par le ministère de la Culture et de la Communication. II co-dirige la série "Les industries de la culture et de la communication", dans la collection "Questions contemporaines », L'Harmattan. 


\title{
et technologies d'information et de communication
}

\begin{abstract}
Résumé
Cet article vise à questionner les relations complexes entre les industries et l'économie créatives, d'un côté, et les Tic, de l'autre. L'interrogation porte également sur les notions. En quoi les débats et les enjeux que suscitent ces notions sont-ils liés, complémentaires ou concurrents? En somme, face aux Tic, de quelles nouveautés - ou continuités- sont porteuses les thématiques des industries et de l'économie créatives? Premièrement, si ces thématiques apparaissent dans les années 1990 au Royaume-Uni en continuité avec les enjeux soulevés par les Tic, elles mettent l'accent sur la défense des droits de la propriété intellectuelle et le rôle central de ces derniers dans l'économie des anciens pays industriels. Deuxièmement, l'analyse des discours sociaux sur les industries et à l'économie créatives montre qu'ils sont porteurs d'une analyse spécifique sur les enjeux de la créativité dans les sociétés et les économiques contemporaines. Troisièmement, la notion d'industries créatives interroge de manière originale les processus de marchandisation et d'industrialisation à l'œuvre au sein des industries de la culture.
\end{abstract}

Mots-clés : Industries créatives, économie créatives, industries culturelles, industries de la communication, technologies d'information et de communication.

\begin{abstract}
This article aims at questioning the complex relations between the creative industries and the creative economy, on one side, and ICT, of the other one. The interrogation also concerns the notions. In what the debates and the stakes, which arouse these notions, are they connected, complementary or rival? As a matter of fact, of what novelties - or continuities are carriers the themes of the creative industries and economy? In the first place, if these themes appear in the 1990s in the United Kingdom in continuity with the stakes raised by ICT, they emphasize the promotion of intellectual property rights and their central role in the economy of the old industrial nations. Secondly, the analysis of the social speeches on the creative industries shows that they are carriers of a specific analysis on the stakes in the creativity in contemporary societies and economic. Thirdly, the notion of
\end{abstract}


Philippe BOUQUILLION

creative industries questions, in a original way, the processes of industrialization within the cultural industries.

Keywords: Creative industries, creative economy, cultural industries, communication industries, information technology and communication.

\section{Resumen}

Este artículo pretende interrogar las relaciones complejas entre las industrias creativas y la economía creativa, de un lado, y Tecnología de Información y de Comunicación, de la otra. La interrogación también se refiere en las nociones. ¿ En qué los debates y las puestas que suscitan estas nociones son complementarios o competidores? ¿ En suma, frente a Tic, de cuáles novedades - o continuidades son portadoras las temáticas de las industrias creativas y de la economía creativa? Primero, si estas temáticas aparecen en los años 1990 en el Reino unido en continuidad con las puestas indignadas por Tic, ponen el énfasis en la defensa de los derechos de la propiedad intelectual y el papel central de estos últimos en la economía de los antiguos países industriales. En segundo lugar, el análisis de los discursos sociales las industrias creativas y a la economía creativa muestra que son portadores de un análisis específico sobre las puestas de la creatividad en las sociedades y económicos contemporáneas. En tercer lugar, la noción de industrias creativas interroga los procesos de industrialización quiénes están en proceso en el seno de las industrias cultural.

Palabras clave: Las industrias creativas, la economía creativa, las industrias culturales, industrias de la comunicación, tecnologías de la información y la comunicación. 
Industries, économie créatives

et technologies d'information et de communication

\section{Introduction}

Les notions d'industries créatives et d'économie créatives sont encore peu présentes en France dans les discours officiels. En revanche, ces thématiques connaissent un succès certain en dehors de l'hexagone, en Europe ou en Australie, mais aussi, quoique dans une moindre mesure, en Amérique du Nord, ainsi que dans des pays dits émergents, notamment en Chine et en Inde. Différentes institutions internationales, en particulier l'United Nation Educational, Scientific and Cultural Organization (UNESCO) ou la Conférence des Nations unies sur le commerce et le développement (CNUCED s'en sont emparées. Ces activités sont alors décrites comme des axes importants de développement. De même, dans les anciens pays industrialisés, pays où le coût de la main d'œuvre est élevé, les industries créatives sont envisagées comme un vecteur essentiel de "sortie de crise". Elles constitueraient un nouveau domaine de spécialisation économique dans le cadre d'une économie mondialisée. Alors que les activités manufacturières issues de la première et de la seconde révolutions industrielles se délocaliseraient en direction des pays émergents, les activités qui reposent sur l'« intelligence ", les «idées " et la "créativité » pourraient rester l'apanage des anciens pays industrialisés.

Les notions d'industries créatives et d'économie créatives sont difficiles à cerner. II est généralement affirmé que diverses activités pourraient être regroupées dans la même catégorie, les "industries créatives ", au motif que la création jouerait un rôle central dans leur production et leur valorisation. Liées à des savoirs et des savoir-faire complexes, supposément spécifiques à un territoire donné, ces activités présenteraient un fort ancrage territorial, produiraient une importante valeur ajoutée et, enfin, seraient hautement créatrices d'emplois. Telle est, schématiquement résumée, la proposition centrale sur laquelle repose la notion d'industries créatives.

La notion d'économie créative, quant à elle, désigne l'extension, à la quasi totalité de l'économie, des procès socio-économiques à l'œuvre, notamment d'organisation du travail ou des modalités de création, dans les industries créatives. David Throsby (2001), un économiste, va ainsi décrire l'économie créative comme une suite de cerces concentriques. Au cœur se trouvent les arts et les industries créatives puis, dans les cercles qui suivent, sont placées les autres activités en fonction du rapport, plus ou mois important, qu'elles entretiennent avec la créativité. Cette représentation va connaître un très grand succès, en particulier dans les définitions officielles des industries créatives qui se succèdent depuis le début des années 2000. 
Philippe BOUQUILLION

Pourquoi les notions d'industries et d'économies créatives connaissent-elles un succès si important sur le plan international depuis le milieu des années 2000 ? Comment et pourquoi ces notions se sont-elles frayées un chemin jusqu'à arriver à l'agenda d'institutions, notamment l'Union Européenne, les Nations Unies ou un ensemble de gouvernements à travers le monde, dans un contexte où la concurrence avec d'autres notions et d'autres idéologies est très forte?

En effet, des notions, ou des propositions idéologiques antérieures, par exemple la "société de l'information", la "société de la connaissance » et, plus encore, l' "économie immatérielle" - toutes clairement liées aux technologies d'information et de communication (Tic)- et toutes aussi floues que les notions d'industries et d'économie créatives auraient pu accueillir les discours liés à la créativité. Par exemple, Maurice Lévy et Jean-Pierre Jouyet, défenseurs de l'économie immatérielle, affirment, dans leur rapport au ministre de l'Economie et des Finances en mars 2006, que les «idées » forment un " capital productif intangible » primordial : «C'est désormais la capacité à innover, à créer, des concepts et à produire des idées qui est devenue l'avantage compétitif essentiel. Au capital matériel a succédé, dans les critères essentiels du dynamisme économique, le capital immatériel. " (Lévy, Jouyet, 2006)

L'ampleur du déploiement des discours relatif à la créativité, aux industries et à l'économie créatives est d'autant plus surprenante que les activités liées aux Tic sont d'une taille économique et financière bien supérieure à celle des activités culturelles. Une comparaison, même rudimentaire, entre, d'un côté, les industries de la culture ${ }^{1}$, y compris élargies au design, aux jeux et, d'un autre côté, les industries de la communication qui rassemblent diverses activités étroitement liées aux Tic et, en particulier, les industries du Web, des télécommunications et des matériels électroniques grand public montrent que les secondes sont beaucoup plus importantes sur les plans économiques et financiers. Les tailles des marchés et celles des principaux acteurs industriels au regard des chiffres d'affaires, des taux de bénéfices et, plus encore, de la valorisation boursière sont sans commune mesure à l'avantage des industries de la communication.

Face à une telle disproportion, on pourrait estimer que les enjeux sociaux suscités par la culture ou la "créativité culturelle ou esthétique » sont vraisemblablement moindres que ceux qui sont générés par les Tic. Aussi, le

\footnotetext{
${ }^{1}$ Elles comprennent les filières du livre, de la presse et de l'information, de la musique enregistrée et du cinéma et de l'audiovisuel.
} 
Industries, économie créatives

et technologies d'information et de communication

développement d'importants discours, d'actions et de politiques de la part des institutions publiques et politiques, des acteurs socio-économiques mais aussi de la part d'experts, de consultants ou de chercheurs est certainement le signe que des enjeux spécifiques sont à l'œuvre. Et, dans le même temps, il apparaît évident que les Tic jouent un rôle essentiel dans ces discours, actions et politiques relatives aux industries et l'économie créatives. II est fréquemment souligné qu'à travers la numérisation de la " chaîne " dans les industries de la culture, depuis la création jusqu'à la valorisation ou à la consommation, l'impact des Tic est très fort. De surcroît, les Tic étant aussi des industries, que l'on peut nommer les industries de la communication, c'est-à-dire les industries du Web, des télécommunication ou de la fabrication de matériels électroniques, les articulations entre, d'un côté, les industries de la culture même élargies au design, aux jeux ou à la mode et, de l'autre les industries de la communication, sont clairement au cœur de notre sujet.

Afin de mieux comprendre les relations complexes entre les industries et l'économie créatives, d'un côté, et les Tic, de l'autre, et en quoi ces propositions et les débats qu'elles occasionnent sont liés, complémentaires ou concurrents, en quoi les enjeux sont spécifiques ou semblables, nous pourrons procéder en trois temps. Nous pourrons alors présenter les différents articles de cette livraison de la revue Tic et société.

Tout d'abord, il convient d'étudier les premiers développements des notions d'industries et d'économie créatives, ensuite, de s'intéresser aux discours sociaux, notamment aux rapports officiels en Europe consacrés à ces thèmes et, enfin, d'aborder les enjeux scientifiques posés par ces deux notions.

\section{L'émergence des notions d'industries et d'économie créatives}

Deux temps peuvent être distingués dans l'émergence des notions d'industries et d'économie créatives qui se déroule entièrement au RoyaumeUni.

\subsection{Le temps de l'émergence}

Durant la première période, les deux notions se développent en parallèle et elles apparaissent comme les héritières directes des débats et propositions liées aux Tic. Toutefois, des définitions des industries créatives sont progressivement construites en lien avec des enjeux industriels spécifiques. 


\section{Philippe BOUQUILLION}

C'est au Royaume-Uni que la notion d'industries créatives apparaît durant les années 1990 et tend à substituer, du moins dans les rapports officiels, à celle d'industries culturelles. Les notions d'industries et d'économie créatives se sont construites conjointement. Selon Nicolas Garnham (2001), les racines de cette thématique sont liées aux déploiements des perspectives néo-libérales dans le Royaume-Uni des décennies 1980 et 1990 dans une articulation avec le développement des Tic. Nicolas Garnham s'intéresse, en particulier, à l'évolution de la position officielle du parti travailliste sur ces questions entre le début des années 1980 et l'arrivée au pouvoir du New Labour, en 1997. II montre que ce nouveau paradigme socio-économique est alors basé autour des technologies de communication numériques : la production ne serait plus essentiellement d'ordre matériel mais elle relèverait de l'intangible. Cette conception est redevable aux think-tanks des conservateurs. En 1983, le gouvernement de Margaret Thatcher avait en effet commandé un rapport officiel Making a business of Information. Les industries liées aux Tic et à la créativité sont présentées comme le secteur le plus porteur de croissance. Le rapport souligne, en outre, que les deux priorités de l'action publique doivent être, d'une part, de promouvoir la formation et l'efficacité des travailleurs créatifs et, d'autre part, de faire observer les lois sur la propriété intellectuelle, maillon clef de la valorisation.

De même, les agences de communication, et les consultants britanniques, jouent un rôle fondamental dans l'émergence de ces notions. Le terme « villes créatives " a été créé ou, du moins, installé dans le discours de l'aménagement urbain international à la suite des propositions de l'agence Comedia et de son emblématique fondateur, Charles Landry. Des auteurs tel Richard Florida ont ensuite cautionné cette notion, l'ont développée et construite sur le plan théorique. En 2002, Florida affirme dans The Rise of the Creative Class que le dynamisme économique et social d'un territoire est lié à la présence d'artistes, d'écrivains, d'acteurs, de designers ou d'architectes, autant qu'à celle de scientifiques, ingénieurs et intellectuels et que, par conséquent, les villes devraient se doter des équipements et infrastructures les mieux à même d'attirer ces types d'habitants. Dans les trois « $T$ » proposés par Richard Florida figure la « technologie », outre le « talent » et la « tolérance ». Depuis, cette notion s'est largement répandue, et notamment dans les pays émergents ou en développement. En avril 2008, La CNUCED a publié un document tentant de mesurer le degré de développement de l'économie créative dans le monde, Creative Economy Report 2008. The Challenge of assessing the creative economy: towards informed policy making. 
Industries, économie créatives

et technologies d'information et de communication

Pour autant, les notions d'industries créatives et d'économies créatives sont à distinguer afin de comprendre les enjeux non réductibles aux Tic posés par la question des industries créatives. En effet, la perspective centrale, qui consiste à miser sur des produits issus d' "idées ", implique qu'il soit possible de protéger juridiquement ces idées lorsqu'elles deviennent des produits commercialisables. Ainsi, la question des industries créatives est inséparable d'une volonté de défendre les droits de la propriété intellectuelle et, même, de les étendre à des domaines qui auparavant ne pouvaient pas en bénéficier. Gaëtan Tremblay écrit : "L'inclusion dans un même univers des industries culturelles et d'autres industries comme le software et le design présente un double avantage. D'une part, il permet à toutes les activités visées de bénéficier du prestige qui auréole le travail des artistes et, d'autre part, d'afficher un volume d'affaires et un taux de croissance exceptionnels, attribuables surtout au domaine du software et des jeux vidéo. » (Tremblay, 2008, p.76). II note au sujet de l'extension internationale du thème des industries créatives : "Par-delà les bons sentiments, cette initiative a également pour objectif d'étendre et de renforcer la reconnaissance de la propriété intellectuelle et d'inciter les différents ordres de gouvernement à faire respecter le paiement des droits d'auteur ou de propriété industrielle. Une stratégie d'inclusion pour faire respecter les règles du jeu, y compris par les pays qui reproduisent plus qu'ils ne créent. » $(2008$, p.80)

Ainsi, c'est dans la perspective de la défense des droits de la propriété intellectuelle, qu'il faut comprendre les tentatives successives de définition du champ des industries créatives. Concrètement, en 2001, le ministère britannique de la Culture, des Médias et des Sports, The UK Government Department for Culture, Media and Sport (D.C.M.S.), définit ainsi les industries créatives : " those industries which have their origin in individual creativity, skill and talent and which have a potential for wealth and job creation through the generation and exploitation of intellectual property ». Cette définition va servir de modèle pour la réflexion des autres pays. Trois ans plus tard, et en continuité, les industries créatives sont définies comme, "toute activité produisant des produits symboliques avec une forte dépendance sur la propriété intellectuelle et pour un marché aussi large que possible" (CNUCED, 2004) Elles sont présentées comme étant une solution à la crise dans les pays où la main d'oeuvre est onéreuse mais "performante ". De même, une publication de I'UNESCO, "Comprendre les industries créatives, les statistiques culturelles et les politiques publiques ", présente les tentatives de définition, de mapping, d'établissement de guides de déploiement des industries créatives conduites en Asie, notamment en Chine, en Inde, ainsi qu'en Amérique Latine. II est impossible dans le cadre imparti à cet article de rendre compte de l'ensemble des définitions produites, précisons toutefois qu'en novembre 2010, dans le 
Philippe BOUQUILLION

cadre d'Eurostat, a été arrêtée la définition officielle des industries créatives. Elle reprend le modèle des cercles concentriques de David Throsby.

\subsection{Le temps des remises en question}

Dans une seconde période, qui s'ouvre au milieu des années 2000 au Royaume-Uni, la notion d'industries créatives est vivement remise en cause au profit de la notion d'économie créative. Là encore, les Tic sont au cœur des relations entre les deux notions. Les industries créatives ne sont alors plus considérées comme un secteur à soutenir, mais comme une source d'innovation et de créativité. Elles forment un input pour d'autres secteurs et pour l'économie dans son ensemble (Banks, O'Connor, 2009, p.369). D'ailleurs, avec l'idée de social network market, mise en avant par Justin O'Connor, c'est la notion même d'industrie qui tend à disparaître. Cet auteur veut montrer que ce marché particulier constitue en lui-même le système de production des innovations. II n'est pas un système qui viendrait, de l'extérieur, mettre son empreinte sur les activités culturelles mais il est le dispositif par lequel sont créées les valeurs culturelles, ce qui fait des industries créatives (paradoxalement le terme est encore cité alors qu'elles sont supposées disparaître) le moteur du développement des connaissances, des sociétés et de l'évolution humaine (O'Connor, 2009, pp.387-388). Les industries créatives seraient alors des rapports et des jeux entre agents sur un marché de réseau social où des "nouveautés " sont produites et retenues à des fins de production et de consommation. Le Web 2.0 joue un rôle central dans ce type de marché. O'Connor évoque la figure du consommateur-citoyen créatif. Dans cette perspective, les industries créatives ne produisent pas des biens dont la valeur d'usage serait connue et appréhendable selon les processus à l'œuvre pour les autres biens mais elles produisent de la "nouveauté » (novelty), des biens dont la valeur est incertaine et qui, en fait, est fixée par le réseau social dans lequel ces biens particuliers, créatifs, circulent et "fertilisent " l'ensemble de l'économie et de la société. Cette approche va plus loin que la volonté de dépassement des industries créatives par l'économie créative puisqu'il s'agit de " fondre » culture et marché. Kate Oakley (2009, p.402), très critique vis-à-vis de ces perspectives, souligne que l'économie créative versus industries créatives correspond à un double mouvement de libéralisation celui de la culture et celui de l'ensemble de l'économie. Et, ce mouvement se produit sous l'égide des réseaux. 
Industries, économie créatives

et technologies d'information et de communication

Au moment où la notion d'industries créatives est remise en cause au Royaume-Uni au profit, de l'économie créative, voire de l' "innovation créative ", elle rencontre un succès certain à l'étranger, comme en témoignent notamment les divers rapports officiels, surtout européens, auxquels nous nous intéresserons maintenant.

\section{Les industries et l'économie créatives dans les rapports officiels en Europe}

À partir du milieu des années 2000, dans de nombreux pays européens, des rapports officiels sur la créativité ou les industries créatives sont publiés. L'Union Européenne elle-même va publier plusieurs documents. Nous choisissons de les évoquer ici parce que tous posent la même question, celle de la place de la création, de la créativité et des industries créatives dans les pays où le coût de la main d'œuvre est élevé. L'étude de ces rapports ${ }^{2}$ a été conduite dans le cadre d'une recherche menée pour le compte du Département des Etudes, de la Prospective et des Statistiques du ministère de la Culture et de la communication. (Bouquillion, Le Corf, 2010) II apparaît qu'au sein des rapports officiels en Europe, en continuité avec les discours promus au Royaume-Uni, deux visions idéaltypiques de la créativité, des industries créatives et de l'économie créative peuvent être distinguées.

L'une est étroite, elle place l'acte créatif dans le domaine esthétique et culturel au centre des industries créatives, tandis que l'autre, beaucoup plus

\footnotetext{
${ }^{2}$ Les discours de 5 rapports nationaux (Allemagne, Bulgarie, Italie, Pays-Bas, Royaume-Uni), d'un rapport international (Pays Nordiques), ainsi que les discours du rapport de l'Union européenne et du Canada ont été analysés. Les références sont les suivantes: Conférence canadienne des arts. De l'économie à l'écologie: Un cadre stratégique pour la main d'œuvre créative, 2009 ; Department for Culture, Media and Sport, UK. Creative Britain: New Talents for the New Economy, 2008 ; Dutch Ministry of Economic Affairs et Ministry of Education, Culture and Science. Culture \& Economy. Our Creative potential, 2005 ; European Commission. The economy of culture in Europe, 2006 ; Federal Ministry of Economics and Technology and Federal Government Commissioner for Culture and the Media. Culture and Creative Industries in Germany, Research Report, 2009 ; KEA, study for European Commission, The impact of culture on creativity, 2009 ; Nordic Innovation Centre. Creative economy green paper for the Nordic region, 2007 ; Santagata, Walter, White paper on creativity: Towards an Italian model of development, 2009 ; World Intellectual Property Organization, Ministry of Culture of the Republic of Bulgaria. Wipo study of the economic contribution of the copyrightbased industries in Bulgaria, 2007.
} 
Philippe BOUQUILLION

large, s'intéresse à la diffusion de la créativité et de l'innovation dans l'économie et la société. D'un côté, l'accent est mis sur l'existence et le développement d'un "secteur" (le terme est ici employé dans son sens commun) des industries créatives, c'est-à-dire d'un ensemble d'activités industrielles qui, au-delà de leurs différences, présenteraient suffisamment de points communs pour être reconnues comme un secteur et, à ce titre, susceptibles d'intéresser les politiques publiques. De l'autre côté, est mise en avant l'émergence de l' " économe créative ", qui correspond à l'importance de la création et/ou de la créativité dans la plupart des domaines de l'activité économique, du moins, dans les pays dits développés où le coût de la main d'œuvre est élevé.

Les rapports officiels empruntent tous à l'une et à l'autre. Mais leur centre de gravité peut pencher plutôt vers l'une de ces conceptions. Les références à la notion de réseau, qui parfois évoquent les réseaux sociaux ou les infrastructures tels les réseaux Internet, et aux Tic sont plus présentes dans la vision large que dans la représentation étroite des industries créatives.

Les thématiques des industries et de l'économie créatives permettent de mettre en avant des représentations assez précises et, peut-être, plus précises que celles liées aux notions de la société de l'information ou de la connaissance, du devenir de l' "industrie ", des territoires et des rôles de l'Etat dans l'économie et la société.

\subsection{Une vision industrielle}

Deux visions de l'industrie sont présentes dans les rapports. Ni l'une ni l'autre ne s'enferment dans l'opposition entre industries et services.

Premièrement, les industries créatives peuvent être envisagées essentiellement à l'aune de l'économie créative. Dans les Pays Nordiques, ou au Royaume-Uni, les références à la définition large, à l'économie créative, sont très importantes. Les industries créatives tendent à se confondre avec l'économie créative. La représentation qui est développée est celle d'un continuum qui part des activités créatives " centrales » ou du " cœur créatif » qui atteint l'économie créative et, au-delà, l'ensemble de l'économie. Toute l'économie peut être concernée dans la mesure où les activités économiques dans les pays développés tendent à devenir créatives. Dans cette perspective, la référence aux classes créatives, aux clusters créatifs et aux droits de propriété est alors centrale. Cette perspective présente deux grands avantages idéologiques et politiques d'inspiration nettement néo-libérale. 
Industries, économie créatives

et technologies d'information et de communication

En premier lieu, elle permet le gommage des conflits sociaux. L'antagonisme entre les ménages et les entreprises pour le partage de la valeur ajoutée est occulté, tout comme les antagonismes de classes.

En second lieu, le passage à une logique d'économie immatérielle favorise une gestion plus libérale de l'ensemble de l'économie à travers une gestion plus libérale de la culture et des domaines créatifs. Le centre de gravité des économies où le coût de la main d'œuvre est élevé passerait de la production de biens tangibles à une économie de l'immatériel. À cette occasion, un nouveau facteur de production autour de la créativité s'affirmerait. II est donc nécessaire de favoriser un esprit d'entreprise au sein des travailleurs créatifs et des entreprises du secteur. II s'agit notamment de transformer les «idées » en biens tangibles ou, du moins, en produits commercialisables. De même, la figure de l'artiste et du créateur ignorant des réalités économiques et commerciales est fréquemment abordée. Dans cette perspective, il convient d'agir en favorisant une meilleure connaissance des dispositifs de la propriété intellectuelle, des mécanismes financiers et une plus grande connaissance des techniques de gestion.

Deuxièmement, est également présente une analyse fine des caractéristiques industrielles des industries créatives. En quoi sont-elles des industries? Et, en quoi sont-elles des industries spécifiques? Plusieurs éléments sont mis en avant.

En premier lieu, les industries créatives produiraient des biens symboliques faiblement substituables. Dans cette perspective, de nombreux produits dans les économies contemporaines deviennent des produits dont la valeur serait moins «fonctionnelle » que «symbolique ». II est notamment souligné que les biens dont la valeur est essentiellement symbolique peuvent être vendus à des prix relativement élevés, déconnectés des coûts de production et qu'ils offrent une expérience unique au consommateur. Les droits de propriété doivent donc être protégés afin de garantir le maintien de cette position aux mains des acteurs qui ont pu les acquérir. La dimension libérale cohabite ainsi avec le respect de droits de propriété et, ainsi, avec un encadrement du marché.

En second lieu, les industries créatives sont reconnues comme étant hétérogènes. C'est sans doute le rapport allemand qui exprime le mieux l'idée que les diverses "branches » des industries de la culture et créatives sont très hétérogènes et qu'il convient de favoriser des rapprochements.

En troisième lieu, les industries créatives sont décrites comme des activités «transectorielles». Cette dimension ferait des industries une sorte de « courroie de transmission » de la créativité en direction des autres secteurs de 
Philippe BOUQUILLION

l'économie. Le design joue un rôle particulier à cet égard. II est à la fois un secteur en soi et un «diffuseur » de créativité. En effet, le design contribue à organiser les idées selon de nouvelles architectures. De même, il aide à la décision, à la prise de conscience collective, notamment par la représentation graphique des enjeux sociaux et il participe à la « diffusion » des idées.

En quatrième lieu, au-delà de leur hétérogénéité et des seules définitions par secteurs ou branches, les rapports officiels tentent de définir les caractéristiques socio-économiques communes et spécifiques aux diverses industries créatives. C'est encore le rapport allemand qui produit la réflexion la plus approfondie. Dépassant très largement les constats faits par les Britanniques, à la fin des années 1990, du rôle des idées et de la nécessité de les protéger par un renforcement des droits de la propriété intellectuelle, le rapport officiel allemand ébauche une théorie socio-économique des industries créatives. Quatre caractéristiques sont mises en avant : la dimension marchande ; la spécificité de l' " acte créatif »; la dimension esthétique ; l'articulation entre firmes grandes, moyennes et petites comme source de flexibilité et de gestion des risques. C'est certainement ce quatrième point qui est le plus original. II est affirmé que les entreprises créatives seraient en mesure, plus que des firmes relevant d'autres domaines, de se remettre en question, de transformer leurs stratégies, de faire preuve de flexibilité. Par ailleurs, une articulation complexe entre grandes, moyennes, petites et très petites (entreprises individuelles) serait à l'œuvre. Ces articulations permettraient de gérer les risques liés à la création en particulier : le caractère incertain de la demande et la difficulté à planifier les activités.

\subsection{Une vision territoriale}

La créativité devient une thématique centrale dans le renouvellement du marketing territorial. Tous les rapports abordent le thème des territoires dits créatifs. Néanmoins, il convient de noter que la notion de territoire créatif est concurrente de la notion de territoires 2.0. Avec cette dernière notion, la créativité n'est pas seulement une affaire d'industriels ou d'institutions, mais elle concerne aussi les citoyens ou les habitants, considérés comme la principale source de créativité. Charles Landry affirme, à cet égard, que la ville créative est un espace où des habitants appartenant à différents univers sociaux et catégories socio-professionnelles se rencontrent, ce qui favorise l'épanouissement de nouvelles idées permettant d'améliorer la qualité de vie, de travail et de loisirs. Dès lors, l'alternative suivante se pose : soit l'action publique soutient un secteur limité, les industries créatives, considéré comme clef de voûte de la nouvelle division du travail, soit l'accent est mis sur le développement des processus de créativité dans des domaines d'activité 
Industries, économie créatives

et technologies d'information et de communication

différents et assez nombreux. Or, alors que l'accent est mis sur la participation citoyenne dans les projets de territoires 2.0, cet objectif se révèle potentiellement contradictoire avec les propositions de politique publique qui accompagnent les projets de développement des industries créatives. En effet, plutôt que de soutenir des acteurs industriels, il s'agit, selon les discours sur "l'explosion de la créativité ", de donner les moyens aux citoyens, grâce au numérique et à la logique dite 2.0, de contribuer au développement urbain. De surcroît, plutôt que d'insister sur les activités culturelles ou à dimension culturelle, la priorité est donnée aux Tic, au numérique.

Dans les rapports, les deux notions sont présentes. Lorsqu'ils insistent plutôt sur la dimension créative des territoires, les rapports mettent en avant trois éléments.

Premièrement, ces territoires doivent être attractifs pour des entrepreneurs en quête d'implantation mais aussi pour des touristes.

Deuxièmement, les industries et l'économie créatives permettraient de créer de nombreux emplois, en particulier parce que le modèle du salariat n'est pas dominant en leur sein, et ces emplois resteraient ancrés territorialement. Les travailleurs créatifs seraient d'ailleurs en nombre croissant. La question des travailleurs créatifs est un point essentiel, au cœur de l'intérêt des autorités publiques dans les pays développés pour les industries créatives.

Troisièmement, les territoires créatifs sont présentés tels des territoires de tolérance culturelle et d'ouverture à l'immigration. II s'agit là d'un point relativement nouveau du marketing territorial. Sur fond de discours sur les métissages des cultures et de tolérance, est décite une concurrence internationale des territoires qui passe par une reconnaissance du multiculturalisme.

Notons qu'en France, où les actions effectivement conduites par les collectivités territoriales empruntent à la fois au modèle des territoires créatifs et à celui des territoires 2.0, l'hypothétique développement de politiques territoriales en faveur des industries créatives pourrait être facilité par des modalités d'action bien ancrées au sein des collectivités territoriales.

II s'agit, tout d'abord, de la volonté ancienne de légitimer par des considérations économiques des activités culturelles. Ensuite, la logique de "gouvernance ", très présente dans les discours sur les industries créatives, est déjà à l'œuvre au sein des collectivités territoriales, en particulier lorsque les moyens d'intervention des collectivités sont réduits ou lorsque les actions visées concernent des domaines qui sortent des champs d'action habituels des collectivités territoriales. Dans ces deux situations, les collectivités soutiennent 
Philippe BOUQUILLION

des actions conçues et exécutées par d'autres institutions ou acteurs. Enfin, le marketing territorial est une préoccupation fondamentale des collectivités territoriales, qui ne cesse de s'accentuer avec la mondialisation. La thématique des industries créatives satisfait à cette exigence et, de surcroît, elle est porteuse de valeur de consensus et même de "réconciliation ». Les actions en faveur des industries créatives sont supposées articuler la culture, la participation des habitants à la gouvernance du territoire et la promotion d'activités économiques, qui reposent sur l'intelligence humaine, qui sont respectueuses de l'environnement et qui échapperaient aux menaces de délocalisation. La culture, le travail, le capital et la démocratie ne sont ainsi pas présentés sous l'angle de leurs contradictions et des conflits mais sous celui du renforcement du lien social fondé sur la communication sociale et le développement durable.

\subsection{L'Etat et les industries et l'économie créatives}

La plupart des rapports insistent sur le rôle central des autorités locales. II est vrai que, dans la quasi totalité des pays européens, c'est au niveau local et non pas national que les premières initiatives se sont développées. Les autorités locales et régionales sont à la fois plus proches des réalisations et sont, dans nombre de pays européens, mieux à même juridiquement et budgétairement d'intervenir. Cependant, la rédaction des rapports officiels marque une volonté des échelons nationaux de reprendre la main sur ces questions, au moins partiellement, en tant que coordonnateurs, plus que financeurs. La montée de la thématique des industries créatives dans les classements internationaux des pays et plus seulement des territoires locaux et régionaux peut expliquer le souci des Etats de montrer leur présence et ce d'autant que l'Union européenne prend également des initiatives dans le domaine.

Les divers rapports préconisent la mise en place de diverses mesures de politique publique qui peuvent être regroupées autour de 5 axes.

Premièrement, l'intervention publique doit rester limitée. Outre les contraintes budgétaires et juridiques, le constat est souvent fait que les industries créatives " existent" déjà et fonctionnement hors de l'intervention publique. Les éventuelles mesures d'action publique doivent tenir compte de l'existant, et viser à renforcer les réalisations présentes. Toutefois, il est également remarqué que les "retombées " économiques et sociales des industries créatives seraient plus grandes si des outils judicieux de politique publique étaient mis en œuvre. Aucun vaste plan d'action n'est envisagé, mais l'enjeu est de faciliter l'accès aux dispositifs d'aides publiques existants et d'en créer éventuellement de 
Industries, économie créatives

et technologies d'information et de communication

nouveaux, plus adaptés aux spécificités des industries créatives. En particulier, il convient de faciliter l'accès des entreprises des domaines considérés aux dispositifs d'aide à l'innovation et à la créativité, ainsi qu'au système financier et bancaire. C'est essentiellement parce que la notion d'innovation prend, au sein des industries créatives, un sens très spécifique que ces dernières ont des difficultés à se financer, tant auprès des acteurs financiers que des dispositifs d'aide publique. En particulier, les sommes en jeu ne sont pas comparables avec celles qui sont dépensées pour la recherche et le développement dans les secteurs technologiques. De même, il est rare que des brevets soient déposés. L'innovation réside parfois, dans le domaine des industries créatives, dans la mise en œuvre d'un nouveau modèle d'exploitation, ce qui n'est pas brevetable.

Deuxièmement, la défense des droits de la propriété intellectuelle est au centre de l'action publique. Tous les rapports officiels européens étudiés s'accordent sur ce point. Cela est d'autant plus important que l'activité économique des entreprises, leur capacité à lever des fonds, comme à nouer des collaborations est liée à l'estimation de leur valeur et à la protection de celle-ci. Or, la numérisation vient rebattre les cartes, en particulier avec le piratage. En fait, il nous apparaît que la numérisation et le piratage servent d'arguments en faveur du renforcement de la protection juridique de la propriété intellectuelle. Les rapports insistent sur la nécessité de rémunérer les talents créatifs, surtout, désormais, sur les supports numériques. Le principe de la protection de la propriété intellectuelle, conçu en référence aux anciens supports, doit s'étendre aux nouveaux. Néanmoins, la possibilité et l'opportunité de continuer à appliquer le même modèle de protection des droits de la propriété intellectuelle sont tout de même interrogées. L'application trop rigide de ces principes pourrait nuire à la créativité, en particulier elle risquerait de rendre trop rigides les rapports entre producteurs et auteurs. Les dispositifs de protection doivent aussi avoir pour objectif la diffusion rapide des innovations aux consommateurs, sans décourager les innovateurs en les privant de revenus liés à leurs innovations.

Troisièmement, les politiques culturelles devaient se réorienter vers des politiques en faveur des industries créatives et vers des objectifs plus marchands, favorisant les secteurs industriels et les articulations public/privé.

Quatrièmement, face aux spécificités socio-économiques des industries créatives, lesquelles semblent échapper aux règles socio-économiques courantes, un effort de compréhension et de médiation entre les acteurs du marché et leur environnement institutionnel est nécessaire. Telle est la condition indispensable pour que les retombées économiques et sociales potentielles du 
Philippe BOUQUILLION

développement des industries créatives soient effectivement au rendez-vous, notamment selon le rapport officiel allemand.

Cinquièmement, l'université et l'éducation, décrites comme étant au cœur de l'économie créative, sont invitées à mieux s'articuler avec le monde des entreprises. Au Royaume-Uni, l'éducation n'est pas seulement au service des industries créatives, elle est présentée comme une composante de celles-ci. Elle constitue ainsi une part importante de l'attractivité internationale du Royaume-Uni, comme de son offre de services à l'international.

\section{Les industries et l'économie créatives : les enjeux scientifiques}

Les discours précédemment cités, tant produits au sein de rapports officiels que par des auteurs ou des experts, montrent que les notions d'industries et d'économie créatives sont bien ancrées. Elles sont d'ores et déjà porteuses d'effets de réalité. On observe notamment des transformations des politiques publiques, en particulier des politiques culturelles, des politiques en faveur des droits de la propriété intellectuelle ou du droit du travail. Pour autant, au-delà de cette dimension de prophétie auto-réalisatrice, faut-il prendre au sérieux ces notions? Autrement dit, la notion d'industries créatives - et nous nous cantonnerons ici à celle-ci considérant que la notion d'économie créative renvoie à des considérations macro-sociales qui méritent une interrogation spécifique dépassant le cadre dévolu à la présente contribution- apporte-t-elle des interrogations nouvelles et pertinentes que la notion d'industries culturelles ne soulèverait pas ? Ou faut-il considérer qu'il n'y a là qu'une idéologie complémentaire aux représentations liées à la société de l'information ou de la connaissance ? Philip Schlesinger écrit ainsi : "...the doctrine of creativity is now (in the UK) an animating ideology for so- called digital age... (and) may be seen as the latest attempt to rationalize interdepartmental cooperation, to make effective the flow of business intelligence, to encourage the networking, to bring together dispersed creative clusters and to foster talent 》 (2007, p.387).

Deux axes d'interrogation peuvent concourir à apporter des éléments de réponse. II s'agit, d'une part, de savoir s'il convient d'ajouter de nouveaux "domaines " aux industries de la culture, des secteurs (au sens commun du terme) non culturels mais dits créatifs, ce qui justifierait alors de ne plus faire référence à la notion d'industries culturelles. D'autre part, la question est de 
Industries, économie créatives

et technologies d'information et de communication

savoir si, au sein des industries de la culture, des transformations se produisent, mettant en avant des problématiques spécifiques liées à la création, ce qui là aussi pourrait conduire à faire référence à la notion d'industries créatives.

\subsection{Une possible extension du champ des industries de la \\ culture?}

II convient, d'une part, de s'interroger sur les causes et modalités d'une éventuelle extension du champ des industries de la culture vers les industries créatives et, d'autre part, d'examiner quelles caractéristiques communes pourraient correspondre aux diverses composantes des hypothétiques industries créatives.

Premièrement, rappelons qu'au-delà du Royaume-Uni, et en particulier en Europe continentale, l'émergence et l'affirmation des notions d'économie créative et d'industries créatives et leur mise en parallèle ou en concurrence avec la notion d'industries culturelles sont liées à trois mouvements se développant à l'échelle internationale :

- les libéralisations des secteurs de la culture et de la communication qui favorisent la mondialisation culturelle et ainsi mettent en avant une vision très large de la notion de culture ;

- les articulations entre industries de la culture et industries de la communication, lesquelles conduisent à une perte d'autonomie de l'économie des industries de la culture ;

- et la montée des mouvements de «culturalisation " de l'économie et de « chosification » de la culture.

En premier lieu, les libéralisations sectorielles (télévision, télécommunications), la libéralisation des échanges de services, et la libéralisation financière, outre la remise en cause des missions traditionnelles de l'intervention publique, font des industries de la culture et de la communication un enjeux économique important, tant sur le plan national, que sur le plan international. Les jeux de l'économie-monde de la culture et de la communication s'intensifient, tandis que les enjeux économiques, industriels, culturels, politiques et sociaux s'enchevêtrent de manière complexe. Les questions de culture et de communication prennent alors une place permanente sur les agendas politiques et industriels. Ce faisant, les débats s'élargissent de la seule culture, au sens culturaliste, à un ensemble plus vaste, celui de la création et des produits dits créatifs, c'est-à-dire des produits qui incorporent de 
Philippe BOUQUILLION

la création et dont la valeur est étroitement liée à leur dimension créative. Ainsi, les travaux de certains des théoriciens de la mondialisation culturelle concourent à élargir la notion de culture. Par exemple, l'approche de l'économiste Tyler Cowen (2002), selon laquelle «le commerce culturel augmente le menu de choix disponible pour chacune des sociétés engagées dans l'échange, mais diminue la différence entre menus de choix de ces deux sociétés ", conduit à formuler une définition si large de la culture qu'elle inclut un vaste ensemble de produits supposément créatifs, dont notamment des produits alimentaires. De nouvelles catégories intellectuelles s'affirment, les débats académiques et politiques se déplaçant des industries et de l'économie culturelles vers l'économie et les industries créatives. Dès lors, noyées dans un ensemble très vaste, les productions culturelles ne semblent plus constituer une catégorie propre à justifier la mise en œuvre de politiques publiques spécifiques. La volonté de certains pays, dont la France, de défendre leurs productions nationales en est peu ou prou disqualifiée.

En second lieu, grâce aux libéralisations, mais aussi aux innovations technologiques, en particulier la numérisation, les articulations entre industries de la culture et de la communication sous l'égide des industries créatives s'intensifient. Alors que ce mouvement a connu des échecs importants dans les années 1990, tout se passe comme s'il connaissait une nouvelle actualité depuis le milieu des années 2000. À la faveur de ces articulations, l'économie des industries de la culture devient très liée à celle des industries de la communication.

Tout d'abord, des acteurs des industries de la communication entrent dans l'économie des contenus. Des acteurs en place des industries de la communication, par exemple Apple, Orange, des fournisseurs d'accès à Internet, deviennent des acteurs clés de la diffusion, de la valorisation et même parfois de la création/production des contenus. Des acteurs émergents des industries de la communication, en particulier des pure players du Web, et des sites du Web collaboratif deviennent eux aussi des places centrales de création/diffusion/valorisation/ promotion des contenus.

Ensuite, les modes de création, production, diffusion, valorisation, bien établis parmi les industries de la culture, sont fortement remis en cause. À cette occasion, la tendance qui fait des contenus des «produits joints ", c'est-à-dire en l'occurence des produits liées à la valorisation d'ensemble des acteurs des industries de la communication s'accroît. Avec cette tendance, le mouvement de bipolarisation des productions culturelles s'affirme. La bipolarisation signifie que les productions de contenus s'orientent dans deux voies, soit la production de contenus premium, aux coûts élevés et assumés par les grands acteurs des 
Industries, économie créatives

et technologies d'information et de communication

filières, soit la production, par les acteurs des franges des oligopoles, de contenus à bas coûts. Même si les marchés demeurent de taille différente et que les filières conservent leurs spécificités, les articulations s'intensifient et jouent un rôle important notamment dans les jeux concurrentiels entre acteurs des industries de la communication.

Enfin, des produits des industries de la communication, essentiellement des matériels électroniques grand public, cherchent à se " culturaliser ». Outre, que les contenus prennent une place désormais centrale dans leur valorisation, certains d'entre eux empruntent des traits aux modes de fonctionnement des produits culturels. Ils visent alors à transformer leurs produits en des produits de création. Telle est la politique marketing de certains d'entre eux, dont Apple et ses iPod ou iPhone. Or le propre des produits culturels est d'incorporer du travail artistique, culturel ou intellectuel. C'est bien leur dimension "créative » qui les distingue radicalement des autres produits de l'économie. Leur valeur d'usage est liée à la "qualité » du travail créatif incorporé. La stratégie de ces industriels est donc de suggérer que leurs matériels sont des produits créatifs, non substituables et rapidement obsolètes. Ils tentent ainsi de distinguer leurs produits de ceux des offres concurrentes en évitant une concurrence par les prix. Ils espèrent alors se ménager des taux de marge élevés.

En somme, alors que les enjeux économiques, comme culturels et politiques, soulevés par la culture et la communication sont exacerbés, les limites des industries culturelles deviennent plus floues. Concrètement ces articulations favorisent :

- Une perte d'autonomie économique des produits culturels et de leurs acteurs historiques.

- Un amoindrissement de leur valeur symbolique lorsqu'ils prennent sens par rapport à la valorisation d'autres produits et lorsque des contenus, très différents, notamment «amateurs » et issus des industries de la culture sont mis sur le même plan.

- La remise en cause des dispositifs réglementaires spécifiques, par exemple de dépenses obligatoires dans la production, au nom, d'une part, de l'égalité des positions concurrentielles entre acteurs historiques et «nouveaux » acteurs des contenus et, d'autre part, au nom de l'abondance "créative" liée aux nouveaux médias et supports en développement. 


\section{Philippe BOUQUILLION}

- La « réconciliation » entre économie, culture et communication, selon l'expression promue par Jack Lang, dans le cadre de l'économie créative.

Ainsi, ces articulations entre industries de la culture et industries de la communication suscitent un premier mouvement de «chosification » de la culture et de « culturalisation » de l'économie.

En troisième lieu, ce double mouvement de chosification de la culture et de culturisation de l'économie s'approfondit au fur et à mesure que les contenus culturels et informationnels tendent à s'insérer dans l'économie des produits de consommation. En particulier grâce aux articulations entre Web, Web collaboratif et contenus, les produits culturels et informationnels sont associés à la diffusion/valorisation/promotion de divers produits et services de consommation qu'il s'agisse de produits alimentaires, de vêtements, d'automobiles ou de services financiers. Les contenus peuvent être employés dans des dispositifs destinés à étudier les comportements des consommateurs à partir de corrélations entre leurs consommations culturelles et les autres types de consommation. Ces articulations tendent alors à assimiler les produits de consommation offerts par les marques à des contenus culturels. Pour ne citer des exemples que dans le seul univers du Web collaboratif, tel est l'un des principaux objectifs de la base musicale de Diesel ou des "chaînes "vidéo d'Honda sur le Web 2.0. II s'agit donc du même phénomène de culturisation que celui que nous décrivions plus haut autour d'Apple mais, cette fois-ci, dans des domaines parfaitement étrangers aux industries de la communication. Des auteurs, tels Scott Lash et Celia Lury (2006), insistent sur le double processus de culturisation de l'économie et de chosification de la culture. Dans cette perspective, les créations culturelles deviennent l'archétype du produit dans l'économie contemporaine. Au centre de cette conception est la notion de dépassement de la marchandise par la «marque » (brand), qui se caractérise par un ensemble de relations ; une histoire et une mémoire et une «identité ». Contrairement à la marchandise culturelle, du moins à la représentation qu'en donne Lash et Lurry, la «marque » produit de la différenciation. Les biens culturels ne seraient désormais plus des marchandises «mortes » mais des objets virtuels, qui accèdent à la réalité par le biais de leur transit au sein de l'imaginaire social.L'imaginaire social devient alors le moteur des transformations structurelles de «l'industrie culturelle globale». Ce raisonnement n'est pas sans rappeler la définition du marché produite par James O'Connor et évoquée plus haut. Ces mouvements d'articulation entre culture, communication et produits de consommation favorisent les tendances à l'harmonisation des régimes de propriété. Ils visent également à rendre plus 
Industries, économie créatives

et technologies d'information et de communication

flexible le marché du travail, à externaliser certaines tâches, à réduire le salariat, à déporter les coûts, etc. Les procès à l'œuvre au sein des industries de la culture sont supposés s'étendre dans d'autres secteurs économiques conduisant à représentation de l'économie sur le modèle des cercles concentrique précédemment décrit. Cela correspond d'ailleurs à la perspective du développement de l'économie créative telle qu'elle est présentée dans les discours britanniques et européens.

Deuxièmement, si les frontières des industries de la culture s'élargissent jusqu'à englober certaines des productions des industries de la communication, voire certains des produits de consommation offerts par les marques, alors il serait nécessaire de s'interroger sur l'existence d'éventuelles caractéristiques socio-économiques communes et spécifiques à ces activités ou, du moins, aux activités généralement regroupées au sein des premiers cercles des industries créatives, à savoir les quatre filières des industries de la culture auxquelles s'ajouteraient le design, les jeux vidéo et la mode. Sur la base des travaux relevant des théories des industries culturelles, mais aussi sur la base des travaux de Richard Caves (2000), huit indicateurs permettant d'identifier si les points communs ou les différences ces activités peuvent être proposés.

Le premier est certainement l'incertitude pesant sur la valorisation. Richard Caves en fait, avec d'autres auteurs, mais à sa manière, la caractéristique principale de ces industries. II propose d'ailleurs de "lire » les industries créatives à la lumière de la théorie des contrats. Conclure des contrats permettrait à l'entrepreneur culturel de limiter les risques financiers et de mieux coordonner les procès de production sur un terme relativement long. Fondamentalement, l'incertitude est liée à la nature de «bien symbolique » des produits culturels. L'insertion de travail artistique ou intellectuel limite, d'une part, la possibilité de rationaliser les procès de production et, dans une certaine mesure, de diffuser les produits. D'autre part, cette insertion rend aléatoire la réaction du public face aux produits qui sont considérés comme des "biens d' expérience ". Quelles que soient la qualité des critiques, l'importance des campagnes de promotion, la présence de stars, la réaction du public demeure imprévisible.

Un second indicateur est lié aux coûts. Les coûts unitaires sont très différents selon le type de produits culturels considérés. Cependant, ils présentent, à des degrés divers, trois caractéristiques communes.

Tout d'abord, ils ne sont pas proportionnels aux possibilités de gains. D'une part, parce que leur valeur marchande est liée à leur contenu symbolique. C'est 


\section{Philippe BOUQUILLION}

dans les arts plastiques que cette caractéristique joue le plus, la valeur marchande d'une œuvre étant sans lien avec le coût des matériaux qui la composent. D'autre part, parce que les coûts marginaux sont faibles ou supposés tels. Une fois le produit conçu, un livre par exemple, le coût marginal d'impression et de diffusion est relativement modeste. II l'est d'autant plus que le produit est dématérialisé. Les droits de propriété sont d'autant plus importants que les coûts fixes de création du produit sont importants. Des « rentes» peuvent alors être générées, du moins tant que le produit n'est pas dans le domaine public.

Ensuite, ils sont « irrécupérables », c'est-à-dire que l'acteur économique qui a consenti des dépenses en vue de la production d'un bien ne peut pas récupérer sa mise en cas d'échec. Cette caractéristique accroît donc le risque et elle est d'autant plus forte que les produits sont des prototypes, c'est-à-dire des biens qui n'entrent pas dans une logique de production en série.

Enfin, les acteurs dominants tentent de transférer les coûts vers d'autres intervenants de la chaîne de production. L'organisation des marchés sous la forme d'oligopoles à franges permet ainsi de faire peser une part importante du risque et des coûts de production vers les petits acteurs, qui sont sur les franges. Par ailleurs, dans l'économie éditoriale, le livre et le disque notamment, les auteurs assument une part significative de ces coûts, en particulier la quasi intégralité des coûts de création. Le Web dit collaboratif permet aussi de transférer aux usagers la prise en charge financière de nombre de contenus diffusés sur les supports émergents.

Un troisième indicateur réside dans les modes d'employabilité, de rémunération et dans le statut des travailleurs culturels ou informationnels. D'une part, ils ne sont pas interchangeables, la valeur du produit dépendant de leur personnalité, de leur talent et de leur notoriété. Selon leur notoriété, les travailleurs artistiques et intellectuels peuvent plus ou moins peser sur la répartition des recettes collectées. D'autre part, comme l'écrit Richard Caves (2000, p.3), « creative workers care about their product ». II évoque ici la tension qu'il peut y avoir entre les intervenants au procès de création-production, dont les préoccupations sont principalement d'ordre artistique on intellectuel, et d'autres agents plus intéressés par les perspectives de valorisation.

Un quatrième indicateur est lié au temps. D'une part, les procès de production sont complexes et supposent de coordonner dans le même temps, ou selon une succession bien prévue, la mobilisation de différentes compétences. L'acteur économique principal «loue» les services de prestataires externes, lesquels concourent de façon importante aux procès de production. En outre, le salariat n'est pas la règle. II est concurrencé par le 
Industries, économie créatives

et technologies d'information et de communication

paiement à la tâche, au « cachet » ou par les droits d'auteurs. Le salariat tient une place différente selon le type de filière, la taille des acteurs et leur position dans la filière. D'autre part, la valorisation s'opère dans un temps, soit très court lorsque la durée de vie est éphémère, soit long, lorsque des stratégies de catalogue sont possibles.

Le cinquième indicateur est celui de la reproductibilité. La question du degré de reproductibilité se pose au sein des activités créatives (design, mode/haute gastronomie) comme des activités culturelles (industries de la culture/spectacle vivant). Les critères de l'industrialisation de la formation proposés par Pierre Mœglin (1998), à savoir la «technologisation », l'idéologisation et la rationalisation offrent certainement des clés pour penser l'industrialisation et notamment les «tendances industrialisantes ", selon l'expression de Pierre Mœglin (1998), dans les secteurs qui ne relèvent pas au sens strict des domaines où opère la reproductibilité.

Le sixième indicateur renvoie à la question de la marque. L'intervention de la notion de marque est présente dans les industries de la culture, comme dans les industries créatives, même si ces dernières ont des stratégies plus accentuées. Peut-on comparer, par exemple, d'un côté, les liens entre la haute couture et la vente d'accessoires de mode avec, de l'autre, les logiques de produits dérivés dans les industries de la culture où une même "entité créative ", Mickey par exemple, est déclinée sur une constellation de produits?

Le septième indicateur a à voir avec la question de la créativité et de son " encadrement». II est difficile d'encadrer étroitement la création dans le domaine culturel. Les limites du «marketing amont » ont déjà été soulignées (Miège, 2000), malgré le développement des logiques gestionnaires (Rebillard, 2011). Mais, parallèlement, la question de la créativité dans les industries créatives reste entière. II est nécessaire de bien distinguer la créativité de type scientifique ou technique et la créativité d'ordre artistique ou intellectuel. Or, nombre de discours sur les industries créatives assimilent l'une et l'autre et donnent à croire que des transferts de compétences sont possibles entre artistes, scientifiques, bureaux d'études.

Le huitième indicateur porte sur la question des contributions différenciées des industries de la culture et créatives à l'espace public, aux identités et à leur dimension politique. À l'évidence, les contributions des activités placées au sein des industries créatives à la construction de l'espace public sont fort différenciées. Néanmoins, ces différences sont souvent niées par les discours des promoteurs des industries créatives. En fait, il apparaît qu'au sein même 
Philippe BOUQUILLION

des activités regroupées dans les industries de la culture, les différences sont importantes, par exemple entre la presse et la musique enregistrée. Quoi qu'il en soit, il faut noter que les promoteurs des industries créatives, ou d'ailleurs plutôt des territoires créatifs, notamment lorsqu'ils s'intéressent également aux territoires 2.0, mettent en avant la contribution à la construction de l'espace public, comme du lien social, par des activités tel le design. La notion de " design de services publics » fait ainsi référence à la participation des citoyens, des habitants, ou des usagers d'un service public à la réflexion sur le devenir de la Cité ou d'un service public particulier. Au-delà des références explicites produites par les agents sociaux ou les acteurs socio-économiques à la dimension politique d'une industrie créative, il serait intéressant de s'interroger sur la notion de société à laquelle peuvent renvoyer les modes de fonctionnement de cette industrie. Pierre Mœglin (2007, p.159) lorsqu'il s'intéresse aux modèles des industries culturelles qu'il conçoit comme des idéaltypes considère que chacun de ces modèles est porteur d'une certaine vision de la société. Ainsi, la dimension politique, au sens large du terme, semble incontournable lorsqu'on veut définir la notion d'industries culturelles comme celle d'industries créatives.

\subsection{La création en question}

À la faveur des mouvements d'articulation, précédemment évoqués, entre industries de la culture et industries de la communication, une des questions qui peut se poser est celle d'éventuelles transformations importantes qui mettraient en avant des enjeux liés à la création ou à la nature créative de ces activités et qui pourraient justifier le recours à l'expression industries créatives.

Si cette hypothèse devait être validée, la définition de la notion d'industries créatives qui serait alors donnée serait tout de même assez éloignée de celles des autorités statistiques gouvernementales ou européennes. Ces définitions (voir Bouquillion, Le Corf, 2010) nous semblent plutôt sommaires, floues et discutables. En somme, elles regroupent au sein des industries créatives les activités reposant sur des "idées", transformables en des produits commercialisables qui peuvent être protégés grâce à l'imposition de droits de la propriété intellectuelle.

L'hypothèse que nous présentons ici est tout autre, il s'agit plutôt de considérer qu'une dissociation entre les stades aval et amont des filières est peut-être en train de se produire. Cette dissociation s'accompagnerait du 
Industries, économie créatives

et technologies d'information et de communication

maintien, voire du renforcement paradoxal, de l'importance des activités de création pour les positionnements des acteurs aux deux niveaux opposés de la filière, aval et amont.

Premièrement, au fur et à mesure que les articulations entre industries de la culture et industries de la communication se mettent en œuvre, on peut observer que des acteurs entrent dans l'économie des contenus. Ils peuvent, par exemple, proposer des plateformes agrégeant divers types de contenus culturels ou informationnels, des plateformes sur lesquelles sont distribués divers produits culturels et informationnels issus des filières du livre, de la presse et de l'information, de la musique enregistrée et du cinéma et de l'audiovisuel. Comme nous lavons signalé plus haut, des acteurs aussi différents que Google, Apple ou Orange ne proposent plus seulement des services de moteurs de recherche, des appareils à la vente ou des abonnements à des services de réseaux de connexion mais aussi des contenus. Si leur cœur de métier réside toujours dans ces domaines, les contenus culturels et informationnels sont désormais associés de manière indissociable à leur offre.

II s'agit pour eux d'atteindre deux objectifs. D'une part, ils peuvent avoir besoin de faire apparaître leurs produits comme des produits culturels. On a déjà cité l'exemple des stratégies conduites par Apple de culturisation de ses ordinateurs, téléphones et baladeurs. D'autre part, le fait de disposer, légalement ou illégalement, de contenus les plus exclusifs possibles, qu'ils peuvent associer à leurs offres, est un élément central dans leur positionnement les uns par rapport aux autres. À cet égard, Google a une position assez unique à l'échelle internationale dans un ensemble diversifié de contenus. Apple, à travers l'Apple Store ou iTunes, a également associé des contenus musicaux ou audiovisuels à ses offres d'appareils, sans avoir d'ailleurs à investir de manière importante dans la production. En revanche, un opérateur de télécommunications, tel Orange, soucieux de différencier son offre d'accès à Internet comme de téléphonie vis-à-vis de ses concurrents doit consentir des dépenses significatives pour s'assurer l'exclusivité de diffusion de films en VOD. Orange a dû ainsi créer une filiale de production cinématographique pour contrer Canal Plus qui dispose de l'essentiel des droits de diffusion des films français. L'opérateur historique français des télécommunications doit également acquérir pour des montants significatifs, à l'échelle de l'économie des contenus, des droits de diffusion sportifs. Des réseaux sociaux, par exemple Facebook en particulier à travers l'accord intervenu fin 2010 avec MySpace, cherchent à également à associer leur offre le plus étroitement possible avec des contenus. L'objectif est de se distinguer des autres réseaux sociaux et d'attirer plus d'internautes mais aussi de pouvoir mieux étudier le comportement de ceux-ci et 
Philippe BOUQUILLION

d'être mieux à même de leur proposer des publicités ou des offres commerciales ciblées.

Ainsi, qu'ils investissent ou non dans les contenus, que leur entrée dans les contenus se fasse de manière légale ou illégale (Google Books a numérisé plusieurs millions de titres sans l'autorisation des ayant-droit), ceux-ci n'en demeurent pas moins fort importants pour les acteurs dominants des industries de la communication. Ils tentent notamment d'éviter une concurrence par les prix en diminuant la « substituabilité » de leur offre. L'importance des contenus dans leur économie ne se mesure donc pas au prorata du chiffre d'affaires réalisé directement grâce à ceux-ci, notamment à leur vente à des consommateurs finaux. Par exemple, iTunes n'aurait atteint le stade la rentabilité qu'il y a peu. Les contenus produisent un ensemble complexe d'externalités lorsq'ils sont insérés dans les offres des grands aceurs des industries de la communication. Outre les externalités d'ordre industriel, par exemple la production d'informations marketing permise par l'offre de contenus d'information (news) de la part de Google, la réussite des stratégies de ces acteurs dans les contenus présente des enjeux financiers. Ainsi, la valorisation boursière d'Apple a augmenté très fortement lorsque les usages d'iTunes se sont installés et sont devenus centraux dans l'économie légale de la musique en ligne. Cet acteur industriel a pu alors encore plus facilement développer ses stratégies car il lui a été plus aisé d'augmenter son capital ou de réaliser des emprunts. En somme, bien que les grands acteurs des industries de la communication, lorsqu'ils pénètrent les filières des industries de la culture, se spécialisent plutôt dans l'aval et qu'ils acquièrent une maîtrise très forte de celles-ci, leur position concurrentielle, notamment leurs pouvoirs de marché, sont très liés à des positions qu'ils peuvent détenir ou contrôler dans l'amont, dans la création. Certes, c'est donc la maîtrise de l'aval et leur position d'intermédiaire entre les produits culturels et informationnels et les consommateurs finaux qui expliquent leur position de force vis-à-vis des acteurs des industries de la culture. En revanche, ce sont leurs positions dans l'amont qui concourent à asseoir leurs rapports de force en direction de leurs concurrents directs, les autres grands acteurs des industries de la communication.

Deuxièmement, les industriels de la culture, face à ces stratégies de pénétration de leur domaine par les acteurs des industries de la communication ne risquent-ils pas de devenir de simples fournisseurs de contenus, lesquels serait alors diffusés et valorisés par d'autres acteurs (ceux des industries de la communication) ? Dans cette hypothèse, les acteurs des industries de la culture ne deviennent-ils que de simples prestataires de services? Deux tendances plaident en faveur de cette perspective. 
Industries, économie créatives

et technologies d'information et de communication

En premier lieu, il apparaît que les «nouveaux « supports de diffusion, maîtrisés par les acteurs des industries de la communication ont conquis une place significative dans la diffusion des contenus. La forme de diffusion illégale de diffusion des contenus non libres de droits participe de ce mouvement. Force est de constater que divers acteurs des industries de la communication ont en retirer des avantages, en particulier une plus forte incitation à utiliser leurs services du fait de le présence de ces contenus.

Le recul des ventes de CD face aux diffusions en ligne, qu'elles soient légales ou illégales, l'importance prise par l'information en ligne qui se traduit par une augmentation du marché publicitaire attaché à ce mode diffusion au détriment des marchés publicitaires propres aux formats papier, mais aussi l'importance des usages de vidéos dites collaboratives ou "professionnelles " en constituent autant de témoignages. La filière du livre a été concernée plus tardivement. Néanmoins, le développement du livre électronique est aujourd'hui très net comme l'analyse une étude de l'Idate dont rend compte ZDNet.fr : « À la fin 2010, le marché du livre numérique a décollé partout dans les pays de l'étude mais présente des situations contrastées. Les Etats-Unis deviennent le premier marché au monde avec des ventes d'e-books atteignant 594 millions EUR en 2010. Ils devancent le Japon, marché pionnier, à 527 millions EUR. par le livre électronique " (source: http://www.zdnet.fr/blogs/digiworld/e-book-etat-deslieux-du-marche-39756748.htm,consulté le 9 décembre 2010).

En second lieu, l'importance croissante de ces modes de diffusion et de valorisation conduit à une profonde déstructuration des modèles socioéconomiques, au sens large du terme, autour desquels se sont historiquement constituées les industries de la culture. Cette déstructuration se marque sur trois plans, celui des professionnalités, celui des contenus et des genres et, enfin, celui des modalités de valorisation. Dans le cadre imparti à cette contribution, nous n'évoquerons que ce troisième point. Afin de pouvoir évaluer la portée déstructurante de ces nouveaux modes de diffusion et de valorisation, il faut, au préalable, analyser les stratégies tarifaires dans le domaine des contenus qui sont adoptées par les acteurs des industries de la communication, ainsi que leurs stratégies de valorisation dites indirectes des contenus.

Lorsqu'ils pensent la valorisation directe des contenus, les acteurs des industries de la communication sont placés face à un choix. Soit, ils poussent vers le haut les tarifs des contenus, soit ils poussent à la baisse par rapport aux tarifs en vigueur pratiqués par les acteurs historiques des contenus. Tirer à la baisse les tarifs des contenus présente deux avantages. Le premier avantage attendu par les acteurs des industries de la communication est d'imposer plus facilement leurs offres auprès des consommateurs face aux offres existantes, 


\section{Philippe BOUQUILLION}

notamment celles des acteurs des industries de la culture. Le second avantage est lié à l'objectif central des acteurs des industries de la communication lorsqu'ils pénètrent l'économie des contenus. S'ils cherchent à culturiser leur offre principale ou à la positionner par rapport à celle de leurs concurrents directs dans leur marché principal (les télécommunications, le Web ou les matériels), alors les contenus deviennent pour eux des produits joints. C'est-àdire des produits qui accompagnent la production principale, qui sont liés à celle-ci. Dans cette perspective, l'intérêt des industriels de la communication visà-vis des contenus ne résiderait pas dans les chiffres d'affaires réalisés auprès des consommateurs finaux (ventes de contenus) mais dans les avantages « indirects » (culturisation et renforcement du positionnement).

Avec de telles stratégies, les contenus tendent soit, à être valorisés à l'unité, mais à bas prix unitaire, soit par des systèmes d'abonnement qui tarifie l'accès à un « club » de contenus ou soit, encore, de manière indirecte par la publicité et la collecte d'informations marketing. La place de ce dernier mode de financement devient tout à fait centrale même si les chiffres d'affaires réalisées grâce à la vente d'information par les grands acteurs des industries de la communication ne sont guère rendus publics et sont donc difficiles à évaluer. Notons que ces divers modes de valorisation ne sont pas exclusifs les uns des autres et, dans les faits, ils sont plutôt panachés.

Quoi qu'il en soit, les marges réalisées par les acteurs historiques des contenus ne peuvent sans doute guère se maintenir. Ils en sont doublement handicapés.

D'une part, ils doivent reconsidérer leurs stratégies tarifaires sur les supports en place, tandis que ces faibles perspectives de rentabilité découragent leurs stratégies en direction des supports émergents. À cela s'ajoute bien évidemment qu'ils ne disposent pas des savoir-faire techniques et professionnels pour développer par eux-mêmes certains supports, notamment les matériels et les réseaux. Ils n'ont pas les moyens financiers qui leur permettraient d'investir ces activités. Les acteurs des industries de la communication, en revanche, du moins les plus importants d'entre eux, ont des moyens financiers qui leur permettent d'acquérir des entreprises extérieures possédant des catalogues de contenus ou produisant des contenus originaux.

D'autre part, les acteurs des industries de la culture sont généralement moins bien placés que les acteurs des industries de la communication pour tirer parti des financements indirects propres aux nouveaux supports. En effet, les genres constitués, les savoir-faire liés aux professionnalités en place ont été 
Industries, économie créatives

et technologies d'information et de communication

construits par rapport des contenants précis et ne peuvent pas être transposés sur d'autres supports ou, du moins, lorsqu'ils le sont, cela apparaît relativement artificiel et inadapté. Les travaux coordonnés par Franck Rebillard (2011) ont montré que, dans l'exemple de la presse magazine en ligne, les traditions d'écritures journalistiques propres aux titres papier conduisent ces titres de presse à développer des versions électroniques dont les contenus sont moins bien adaptés pour collecter de la publicité en ligne que ceux produits par les pure players du Web, spécialistes de l'information en ligne. Par ailleurs, les acteurs des industries de la communication sont tendanciellement mieux à mêmes de collecter et à vendre des informations marketing produites grâce à l'insertion des contenus sur des plateformes Web que les acteurs historiques des contenus. Tel est l'objet central de certains des acteurs des industries de la communication, en particulier des réseaux sociaux, des plateformes du Web collaboratif ou des moteurs de recherche. Ils disposent de compétences logicielles indispensables à cette fin. De même, l'insertion des contenus dans ces bases vise principalement à produire de l'information marketing; elle est donc directement conçue dans cette perspective. Elle vise à faciliter les interactions sur les réseaux sociaux ou à aider à la réalisation d'opération de vente ou d'information sur divers produits ou services. Divers contenus collaboratifs ou issus des industries de la culture, ainsi que des contenus textuels, filmiques ou musicaux, se trouvent assemblées dans cet objectif. Les plateformes aux mains des acteurs historiques des contenus ne disposent pas des mêmes avantages. Certes, elles offrent une masse critique de contenus importants, surtout quand les acteurs se sont regroupés, comme par exemple les grands acteurs télévisuels américains au sein de Hulu. Toutefois, c'est là leur seul avantage et celui-ci, dans certains domaines, est parfois contesté. Un acteur tel Google est en mesure dans le domaine du livre, notamment, de mobiliser une masse considérable de contenus. 12 millions d'ouvrages auraient été scannés et seraient diffusés sur Google Books.

\section{Conclusion}

Au terme de cet article, force est de constater que les notions d'industries et d'économie créatives renvoient à des nombreux enjeux hétérogènes. Bien que les définitions de ces notions soient très floues, on comprend que les perspectives visant à développer les industries créatives et à favoriser la "conversion" des économies aux logiques d'économie créative ne se confondent pas avec la promotion des Tic. 


\section{Philippe BOUQUILLION}

Primo, durant leur période d'émergence au Royaume-Uni, ces notions sont les héritières de discours liés aux Tic. Toutefois, elles sont porteuses d'enjeux spécifiques. II s'agit en particulier, à travers leur promotion, de défendre et même d'étendre le champ d'application des droits de la propriété intellectuelle, de penser la place des anciens pays industriels dans l'économie mondialisée et de favoriser une plus grande flexibilité du droit du travail. Lorsque ces notions sont remises en question, toujours au Royaume-Uni, c'est aussi autour d'enjeux non réductibles aux Tic, en particulier la remise en cause des politiques culturelles au profit d'une plus grande marchandisation des activités culturelles.

Secundo, quand ces thématiques se déploient au-delà du Royaume-Uni, diverses représentations industrielles sont construites. Tout d'abord, elles resituent les industries créatives notamment par rapport à leur dimension symbolique, à leur structuration en oligopole à franges, à leur capacité à déployer la créativité dans l'ensemble de l'économie. C'est par rapport à ces réflexions relatives aux spécificités industrielles de ces domaines qu'est pensée l'insertion des Tic dans leur économie. Ensuite, la vision territoriale qui est portée par les rapports marque également la spécificité de la notion de territoires créatifs, différente de celle de territoires 2.0, même si dans les actions effectivement conduites, notamment par des collectivités territoriales françaises, les deux dimensions s'articulent. Enfin, les représentations relatives aux politiques publiques, pour une part, reprennent assez largement des thématiques déjà présentes avec les discours relatifs aux Tic, en particulier la proposition que les politiques publiques doivent aider à construire un cadre favorable pour les acteurs; qu'elles doivent concourir à rapprocher secteur public et secteur privé ; qu'elles doivent être conduites dans une logique dite de "gouvernance » et que l'échelon national a un rôle à jouer entre les niveaux supranationaux et infranationaux. Pour une autre part, des thèmes spécifiques sont développés, en lien avec les discours antérieurement produits par les Britanniques, notamment autour des questions de droits de la propriété intellectuelle; du renouvellement marchand des politiques culturelles; de l'immigration; du rôle de facilitateur de la communication des entreprises créatives que doit jouer l'Etat; de l'éducation et de l'université en tant qu'industries et vecteurs de mobilisation d'une nation autour de la créativité.

Tertio, lorsque sont évoqués, d'une part, les éventuels points communs entre les industries de la culture et les industries créatives et, d'autre part, le poids de la création et le caractère stratégique de celle-ci du fait des articulations croissantes entre les industries de la communication et celles de la culture, la question des Tic est présente. Toutefois, si les débats relatifs à l'élargissement 
Industries, économie créatives

et technologies d'information et de communication

du champ de la culture sont en partie liés aux Tic, notamment parce que le Web est un des facteurs de la mondialisation culturelle. En revanche, les indicateurs des rapprochements entre les divers domaines des industries de la culture et créatives ne sont pour la plupart d'entre eux pas directement liés aux Tic. Du moins, il convient d'examiner précisément comment les Tic interfèrent avec les modalités d'industrialisation et de marchandisation spécifiques aux industries de la culture. En fait, la question qui est posée ici est celle des rapports entre les Tic et les évolutions de l'industrialisation et de la marchandisation. C'est d'ailleurs parce que les Tic sont aussi des industries que les transformations au sein des industries de la culture prennent une si grande ampleur.

Ces indicateurs peuvent en effet être considérés comme des points clefs des transformations de l'industrialisation et de la marchandisation de ces secteurs les enjeux des Tic dépendent des modalités d'industrialisation et de marchandisation spécifiques aux industries de la culture.

\section{Références bibliographiques}

Banks, Mark, O'Connor, Justin, (2009), "After the creative industries ", International Journal of Cultural Policy, Vol. 15, No. 4, November, pp. 365373.

Bouquillion, Philippe, Combès, Yolande, (2007), Les industries de la culture et de la communication en mutation, Paris, L'Harmattan.

Bouquillion, Philippe, Combès, Yolande, (2011), La diversité dans les filières d'industries culturelles, Paris, L'Harmattan.

Bouquillion, Philippe, Le Corf, Jean-Baptiste, (2010), « Les industries créatives et l'économie créative dans les rapports officiels en Europe ", Rapport remis au Département des Etudes, de la Prospective et des Statistiques, ministère de la Culture et de la Communication, Paris, Mai, 43 pages.

Caves, R., Creative Industries: Contracts between Art and Commerce, Cambridge : Harvard University Press, 2000.

Conférence des Nations Unies sur le Commerce et le Développement, (2004), E-commerce and Development Report, (Consulté en ligne $1^{\text {er }}$ décembre 2010, http://www.unctad.org/Templates/Webflyer.asp?intltemID=3356) 
Philippe BOUQUILLION

Conférence des Nations Unies sur le Commerce et le Développement, (2008), "Creative Economy Report. The challenge of assessing the creative economy: Towards informed policy Making ", www.unctad.org/en/docs/ditc20082cer_en.pdf.

Cowen, T., 2002, Creative destruction, how globalization is changing the world's cultures, Princeton, Princeton University Press.

Florida, Richard, (2002), The rise of the creative class. New York, Basic Books.

Garnham, Nicholas, (2001), The UK Cultural Sector, profile a policy issues, in Selwood S. (ed.), London, pp. 445-458

Guesnier, Bernard (2006) : Connaissance, Solidarité, Création. Le Cercle D'or Des Territoires, Paris, L'Harmattan.

Howkins, John, (2001), The creative economy: how people make money from ideas, London, Penguin.

Lash, Scott, Lury, Celia, (2007), Global Cultural Industry, Cambridge, Polity Press Press.

Lévy, M., Jouyet, J.-P., (2006), L'économie de l'immatériel. La croissance de demain, rapport de la Commission sur l'économie de l'immatériel, Paris, Ministère de l'économie, des finances et de l'industrie

Miège, Bernard, (dir.), (1990), Médias et communication en Europe, Grenoble, Presses Universitaires de Grenoble.

Miège, Bernard, (2000), Les industries du contenu face à l'ordre informationnel, Grenoble, Presses Universitaires de Grenoble.

Mœglin, Pierre, «L'approche socio-économique des médias. Problèmes méthodologiques », in Miège, Bernard,, (dir.), 1990, p. 43-59.

Mœglin, Pierre, (dir.), (1998), L'industrialisation de la formation. Etat de la question, Paris, Centre National de Documentation Pédagogique.

Mœglin, Pierre, (2007), “Des modèles socio-économiques en mutation », in Bouquillion, Philippe, Combès, Yolande, pp. 151-162.

Oakley, Kate, (2009), The disappearing arts: creativity and innovation after the creative Industries, International Journal of Cultural Policy, Vol. 15, No. 4, November, pp. 403-413.

O'Connor, Justin, (2009), Creative industries: a new direction?, International Journal of Cultural Policy, Vol. 15, No. 4, November, pp. 387-402. 
Industries, économie créatives

et technologies d'information et de communication

Rebillard, Franck, (dir.), (2011), « Mutations de la presse et de l'information », in Bouquillion, Philippe et Combès, Yolande.

Schlesinger, Philip, (2007), Creativity: from discourse to doctrine?, Screen, Oxford University Press, 48,3, autumn, pp. 377- 387.

Throsby, David, (2001). Economics and culture, Cambridge, Cambridge University Press.

Tremblay, Gaëtan, (2008), Industries culturelles, économie créative et société de l'information, Global Media Journal -- Canadian Edition, Volume 1, Issue 1 , pp. 65-88. 